\title{
Explosives analysis
}

\author{
David S. Moore • John V. Goodpaster
}

Published online: 5 August 2009

(C) Springer-Verlag 2009

It is difficult these days to find a newspaper or magazine that does not mention the latest suicide bombing or improvised explosive device attack somewhere in the world. Explosives are a cheap and easily obtained means to inflict damage and cause terror. They are difficult to detect, especially at a distance, and contain a large amount of chemical energy per mass so that much destructive power can be easily carried and hidden. The past few years have seen a dramatic increase in efforts aimed at explosives detection and explosives analysis, driven mostly by the need to curtail, or at least slow down, this expanding threat [1].

Even before the latest upswing in explosives-related incidents, nearly all known analytical methods had already been investigated for the applicability to the detection of explosives (for example, see reviews by Moore [2, 3], Yinon [4], Urbansky [5], and Steinfeld and Wormhoudt [6]). This special issue is intended to present the most recent advances and the current state of the art in explosives analysis and detection. Most of these methods and approaches are aimed at detecting and identifying explosive materials "left of boom" (meaning before detonation),

D. S. Moore $(\bowtie)$

Shock and Detonation Physics, DE-9, MS-P952,

Los Alamos National Laboratory,

Los Alamos, NM 87545, USA

e-mail: moored@lanl.gov

J. V. Goodpaster

Department of Chemistry and Chemical Biology,

Forensic and Investigative Sciences Program, Indiana University

Purdue University Indianapolis (IUPUI),

402 North Blackford Street, LD 326,

Indianapolis, IN 46202, USA

e-mail: jvgoodpa@iupui.edu allowing effective prevention or minimization of damage. The ultimate goal of these concepts is to enable upstream searches leading to bomb factories or bomb makers. Nevertheless, of equal value are methods of postblast analysis enabling attribution and gathering of concrete evidence for prosecution of perpetrators.

The most difficult areas of research and development are for methods to achieve standoff (many meters distant from the sample) detection of concealed explosives [1]. A variety of nuclear and X-ray methods are used to screen luggage and shipping containers, but these are not appropriate for use on humans. Therefore, a number of new imaging methods, such as millimeter wave and terahertz imaging, are on the rise, as indicated in the review by Leahy-Hoppa et al. and the paper on polarization orientation dependent terahertz spectra by Whitley et al. in this special issue. Hernandez-Rivera et al. show that other vibrational spectroscopies have potential for standoff detection. In this vein, infrared hyperspectral imaging has been demonstrated by Blake et al. to be capable of detecting explosives residues on surfaces at standoff distances.

Other spectroscopic methods are being tested for their standoff detection capabilities, with laser-based methods reviewed by Wallin et al. and other photonics sensor devices reviewed by Willer et al. Particularly active is the field of laser induced breakdown spectroscopy (LIBS). The current status of LIBS efforts is presented in a review by Gottfried and collaborators. A new laser-based standoff detection approach is introduced by Lisa Kelly's article on amplified spontaneous emission.

Another issue critical to the success of trace methods is their signature, meaning the spatiotemporal amount of a given material available for detection. The paper by Kunz et al. is representative of efforts aimed at obtaining such signature information. 
A recent area of interest has been development of methods to detect and analyze peroxide materials. The current trends in detection of peroxide materials are reviewed by Hage et al. Wang and collaborators present a simple and inexpensive amperometric method to detect hydrogen peroxide vapors for security screening applications. The quantitative infrared spectroscopy of hydrogen peroxide vapor, needed for infrared absorption detection methods that could be quite effective for screening applications because of the large vapor pressure of hydrogen peroxide, is given by Johnson et al.

A number of other methods are under investigation. These include new sensing polymer systems that take advantage of Lewis acid-base interactions developed by Bill Trogler and collaborators, a new colorimetric sensor based on chloride anion reactions developed by Sessler et al., and a new bioassay aimed at locating bomb makers from Bell and collaborators. A significant new miniaturization development for handheld ion mobility spectroscopy based sensors is described by Babis et al. Finally, the difficulties encountered for trace characterization and detection of urea nitrate, an exceptionally easy to make improvised explosive, are described by Almog and his collaborators.

We appreciate the venue provided by Analytical and Bioanalytical Chemistry to gather together in one place a collection of papers describing recent developments for analysis and detection of explosives. We hope that the contents of these papers will further stimulate activity enabling the final goal of achieving reliable, accurate, and sensitive methods that will bring a sigh of relief to explosives ordnance disposal technicians, forensic scientists, security operatives, and others whose job it is to ensure the safety and security of civilized people.

\section{References}

1. NRC Board on Chemical Sciences and Technology (2004) Existing and potential standoff explosives detection technologies. NRC/ NAS, Washington

2. Moore DS (2004) Rev Sci Instrum 75:2499-2512
3. Moore DS (2007) Sens Imaging 8:9-38

4. Yinon J (2002) Trends Anal Chem 21:292

5. Urbansky ET (2000) Crit Rev Anal Chem 30:311

6. Steinfeld JI, Wormhoudt J (1998) Annu Rev Phys Chem 49:203

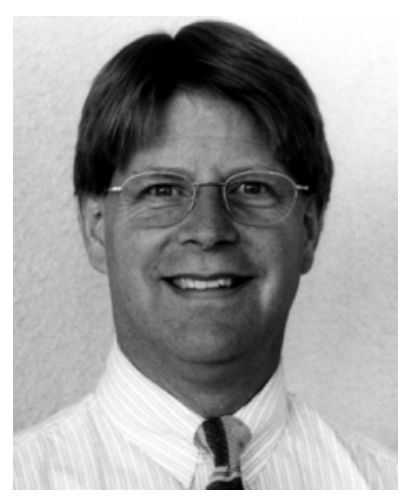

David S. Moore is presently a technical staff member in the Shock and Detonation Physics Group of Los Alamos National Laboratory. His fields of interest cover the application of optimal quantum control methods for condensed phase chemistry (explosive initiation) and dynamic trace detection. He is a Fellow of the American Physical Society (2004), an honor received for breakthroughs in the use of nonlinear optical and ultrafast spectroscopies to understand the behavior of molecules under shock compression. He was a Los Alamos National Laboratory Director Funded Postdoctoral Fellow (1980-1981) and an Alexander von Humboldt Fellow (Essen 1993-1994).

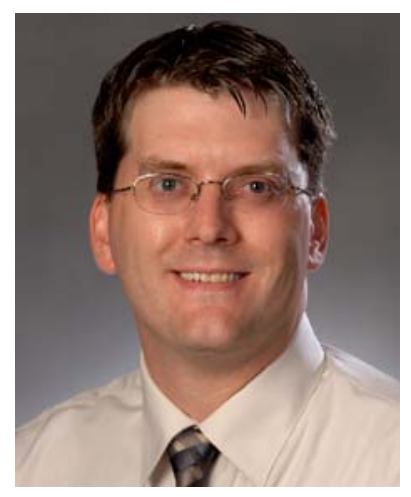

John V. Goodpaster is currently an assistant professor in the Forensic and Investigative Sciences (FIS) Program at Indiana University Purdue University Indianapolis (IUPUI). He teaches in the graduate program in the areas of alcohol and drug analysis as well as trace evidence. Ongoing research in his laboratory includes designing new techniques for explosives analysis as well as instrumental and statistical association of trace evidence. Current collaborations include projects with the Indiana State Police related to explosives, fire debris, and fiber evidence. Lastly, a federally funded project on the chemical compounds sensed by explosive-detecting canines is under way. 